\title{
CICATRISATION CUTANÉE assistée par laser
}

\author{
Serge MORDON \\ INSERM U1189 ONCO THAI, \\ 1 rue du Pr. Calmette, \\ 59037 Lille Cedex \\ serge.mordon@inserm.fr
}

\section{Bases conceptuelles de la cicatrisation cutanée}

La cicatrisation d'une plaie est une procédure de réparation qui conduit à la formation d'un tissu n'ayant plus ses caractéristiques natives [1,2]. En revanche, la régénération est un processus de reconstitution ad integro d'un tissu ou d'un organe voire d'un membre tel qu'on peut l'observer chez la salamandre. La peau fotale humaine lésée peut être complètement régénérée, sans fibrose. Au cours de la vie adulte, le foie est l'un des rares organes dans le corps humain qui
Toute effraction cutanée entraîne une cascade de réactions biologiques dans la peau, dont le but est de rétablir au plus vite et au mieux ses fonctions. La cicatrisation cutanée est un processus biologique complexe, donnant lieu à un remaniement dermique allant de la cicatrice "visible » à des formes de cicatrices hypertrophiques. À l'opposé, la régénération d'un tissu ou d'un organe est la reconstitution ad integro de ce dernier. De récents travaux suggèrent qu'au moyen de lasers générant un effet thermique précisément défini dans la peau, il devient possible d'améliorer le processus de cicatrisation jusqu'à se rapprocher d'une régénération tissulaire. peut se régénérer sans formation de cicatrice. Comment la régénération se produit-elle, quels sont les signaux biologiques qui permettent une régénération tissulaire, pourquoi l'homme, dès la naissance, perd-il cette capacité, autant de questions encore non résolues... Les mammiferes ont cependant conservé une grande partie de la machinerie moléculaire utilisée par des organismes tels que les salamandres, mais leur potentiel de régénération est limité. En partie, cela semble être le résultat de la formation rapide d'un tissu fibreux qui empêche la régénération tissulaire ultérieure.
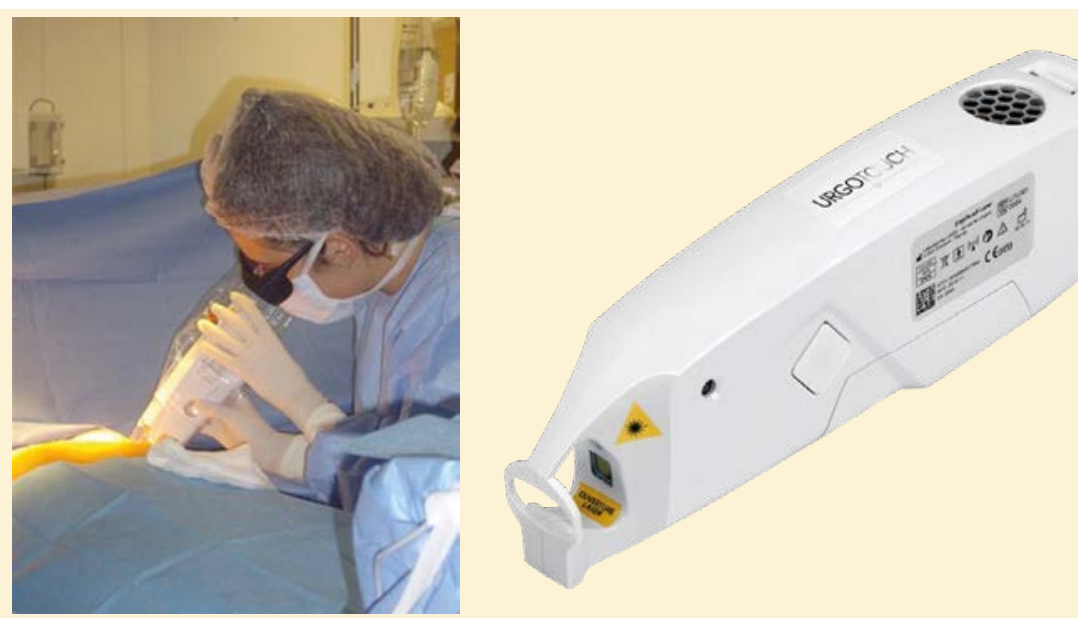

Figure 1. L'irradiation laser est effectuée par le chirurgien immédiatement après la suture à l'aide d'un laser diode $810 \mathrm{~nm}$ portatif.
Manipuler le processus de réparation des plaies chez les mammiferes afin d'obtenir un processus de régénération nécessite probablement la capacité de ralentir le processus de fibrose rapide afin que les cellules multipotentes telles que les cellules souches ou progénitrices puissent à nouveau permettre la régénération tissulaire plutôt que la formation de cicatrices [3]. Différentes équipes ont cherché à bloquer la formation de cicatrices, en particulier en diminuant la production de TGF- $\beta 1$ (ou transforming growth factor beta 1 , une cytokine polypeptidique qui exerce de nombreuses fonctions cellulaires, y compris le contrôle de la croissance cellulaire, la prolifération cellulaire ou la différenciation cellulaire) ou en augmentant la production de TGF- 3 , voire en agissant sur d'autres médiateurs pro-inflammatoires [4]. Bien que d'énormes progrès aient été accomplis dans la délimitation de la myriade de facteurs impliqués dans des conditions normales et pathologiques de la réparation des tissus, ces résultats n'ont pas abouti à des avancées substantielles dans les soins aux patients. En effet, de nombreuses autres cytokines telles que le platelet derived growth factor (PDGF), l'insulin like growth factor (IGF), l'epidermal growth factor (EGF) jouent aussi un 

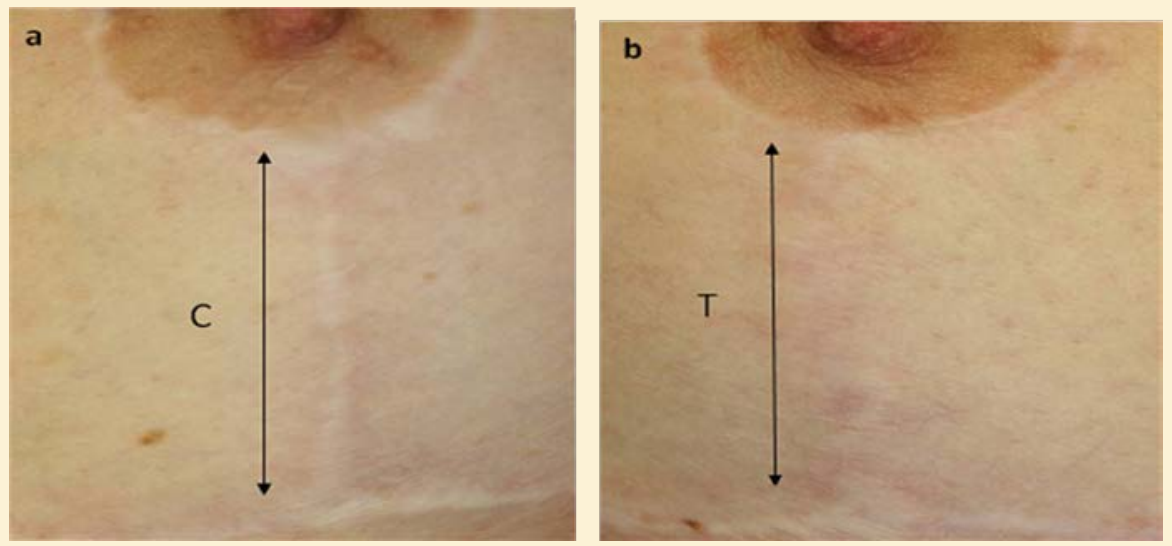

Figure 2. Résultat clinique (sein, cicatrice verticale). (a) Femme (35 ans) non traitée.

(b) Femme (35 ans) traitée par laser. Les parties traitées (T) et contrôlées (C) sont indiquées. rôle et le contrôle d'une seule d'entre elles n'est pas suffisant. Il est cependant apparu que des changements de l'environnement physique (thermique ou mécanique) peuvent modifier le processus de cicatrisation en activant simultanément plusieurs de ces cytokines $[5,6]$.

\section{La technique LASH}

C'est ainsi que dès 2001, l'INSERM à Lille a pu proposer l'utilisation d'un laser thermique pour modifier la réponse cicatricielle. Une étude réalisée sur l'animal avait pu montrer qu'un quasi-processus de régénération de la plaie pouvait être observé [7]. Dans cette étude expérimentale réalisée chez le rat Hairless, où l'animal était son propre contrôle, nous avions pu montrer que des sutures chirurgicales exposées à la longueur d'onde de $815 \mathrm{~nm}$ donnaient des cicatrices nettement moins visibles que les contrôles. L'analyse histologique avait confirmé l'accélération du processus cicatriciel et l'analyse tensiométrique avait montré une meilleure résistance mécanique des plaies traitées par laser par rapport aux contrôles. Cette irradiation laser appliquée dans l'épiderme et le derme entraînait une augmentation modérée de la température tissulaire, provoquant l'activation des protéines de stress (HSP70), qui sont connues pour moduler le processus de réaction inflammatoire et indirectement améliorer la cicatrisation [8].

À l'issue de cette étude, la conception d'un laser spécifiquement dédié à cette application avait pu être initiée en partenariat avec la Société EKKYO, dont les actifs sont désormais détenus par la société Urgo (www.urgotocuh.fr).

Ce laser a pu faire l'objet de plusieurs évaluations cliniques. Une première phase sur 15 patients (juillet 2006-décembre 2007) avait permis d'évaluer l'utilisation de ce laser (efficacité

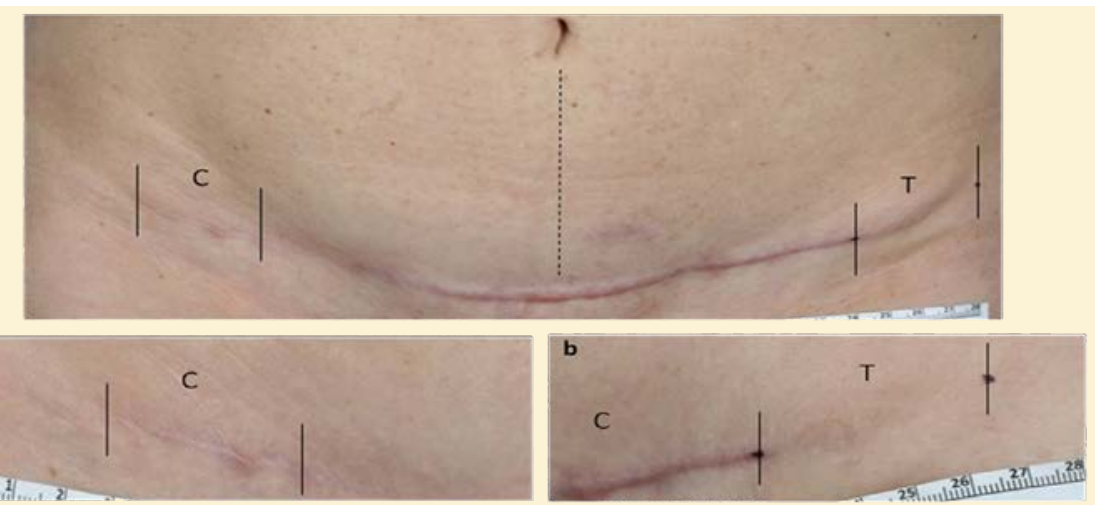

Figure 3. Résultat clinique (abdominoplastie). (a) Femme (47 ans) non traitée. (b) Femme (47 ans) traitée par laser. Les parties traitées (T) et contrôlées (C) sont indiquées [10].

\section{FORMATION CONTINUE}

\author{
Une nécessité \\ pour les entreprises
}

\section{Une mission majeure de I'Institut d'Optique}

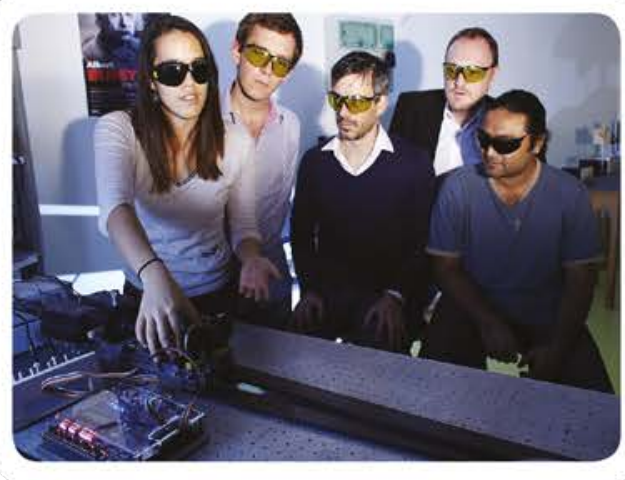

- Au catalogue ou sur-mesure

- Plus de 150 montages pédagogiques

- De l'initiation à la spécialisation

- Formateurs experts

- En anglais ou en français

- En France ou à l'étranger

- Liens forts avec l'industrie

- Infrarouge, optronique

- Instrumentation, composants,

fabrication

- Conception optique

- Imagerie, mesure, communication

- Sources, éclairage, lasers, sécurité

- Mastère Spécialisé ${ }^{\oplus}$ ELS

Systèmes d'Éclairage Embarqué

\section{Nous contacter \\ fc@institutoptique.fr}

Emilie Ericher

+33164533236

Annie Keller

+33164533215 
Patient $n^{\circ} 1$

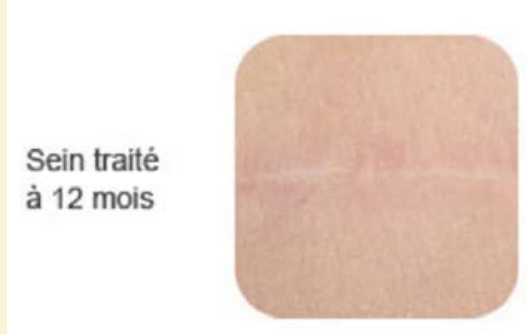

Sein non traité à 12 mois
Patient $n^{\circ} 9$
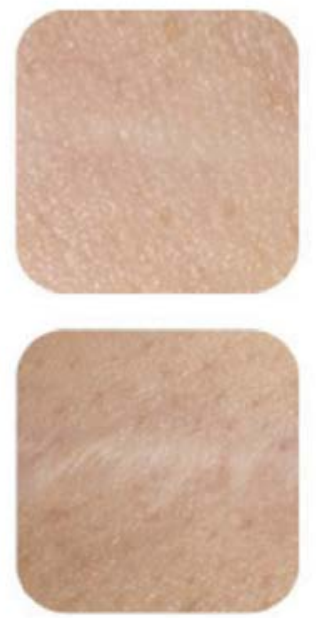

Patient $n^{\circ} 29$
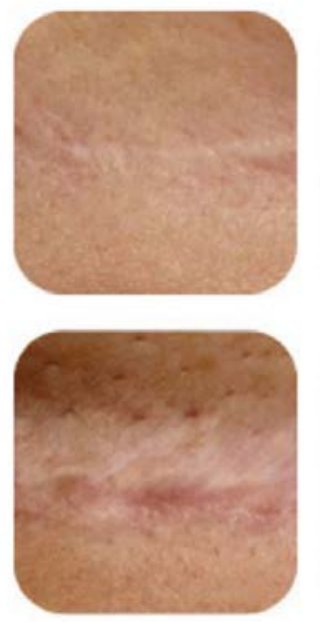

Patient $n^{\circ} 8$
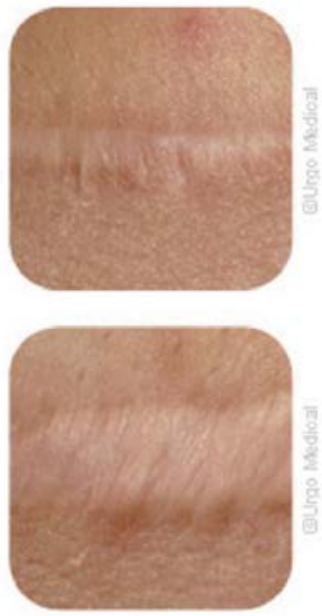

Figure 4.

Exemple de cicatrices observées à 12 mois. et sécurité), et de déterminer les doses efficaces de traitement. L'analyse statistique (test de Wilcoxon), basée sur 11 patients ayant un suivi complet à 1 an, avait mis en évidence des résultats significatifs, à la fois pour le chirurgien $(p=0,046)$ et les patients $(p=0,025)$, les patients se montrant, dans leur globalité, plus satisfaits que le chirurgien $(p=0,020)^{1}$. Aucun effet secondaire n'avait été rapporté [9].

En 2011, nous avons procédé à l'évaluation de différentes longueurs d'onde afin de déterminer celle qui offrait le meilleur compromis entre pénétration tissulaire (absorption par l'eau) et absence de dyschromie (absorption par la mélanine). C'est la longueur d'onde $1210 \mathrm{~nm}$ qui a alors été retenue [11]. L'efficacité et la sécurité de ce dispositif médical ont été testées cliniquement sur 40 patientes (réduction mammaire) lors d'une étude réalisée au sein du service de Chirurgie Plastique et Réparatrice de l'Hôpital de la Conception à Marseille dirigé par le Pr. Casanova [12]. On a ainsi constaté une amélioration moyenne du volume de la cicatrice de $36 \%$. Cinq paramètres

Le seuil observé ( $p$-value, en anglais) peut être calculé et il correspond à la probabilité que le résultat obtenu soit le fruit du hasard: $p<0,05$ signifie donc qu'il y a moins de $5 \%$ de chances que ce résultat ait été obtenu par hasard. ont été pris en compte: la vascularisation, la pigmentation, l'épaisseur, le relief et la surface de la cicatrice.

En conclusion, grâce à un contrôle précis des paramètres lasers, et par conséquent de l'élévation de température au sein del'incision chirurgicale, il est possible d'obtenir un processus de cicatrisation reproductible permettant de réduire considérablementl'aspect cicatriciel généralement observé après l'intervention chirurgicale. La conception originale de ce laser permet une utilisation au bloc chirurgical par les chirurgiens.

\section{POUR EN SAVOIR PLUS}

[1] Rhett JM, et al., Novel therapies for scar reduction and regenerative healing of skin wounds. Trends Biotechnol, 2008. 26 (4): p. 173

[2] Gerbault O, Cicatrisation cutanée. Encycl Méd Chir (Elsevier, Paris), Techniques chirurgicales. Chirurgie plastique reconstructrice et esthétique, 1999. 45-010: p. 19

[3] Gurtner GC, et al., Wound repair and regeneration. Nature, 2008. 453 (7193): p. 314

[4] Ferguson MW, O'Kane S, Scar-free healing: from embryonic mechanisms to adult therapeutic intervention. Philos Trans R Soc Lond B Biol Sci, 2004. 359 (1445): p. 839

[5] Aarabi S, et al., Mechanical load initiates hypertrophic scar formation through decreased cellular apoptosis. FASEB J, 2007. 21 (12): p. 3250

[6] Shamberger RC, Talbot TL, Tipton HW, Thibault LE, Brennan MF, The effect of ultrasonics and thermal treatment on wounds. Plast Reconstr Surg, 1981. 68 (6): p. 860

[7] Capon A, Souil E, Gauthier B, Sumian C, Bachelet M, Buys B, Polla BS, Mordon S, Laser assisted skin closure (LASC) by using a 815 -nm diode-laser system accelerates and improves wound healing. Lasers Surg Med, 2001.28 (2): p. 168

[8] Souil E, Capon A, Mordon S, Dinh-Xuan AT, Polla BS, Bachelet M, Treatment with 815-nm diode laser induces long-lasting expression of $72-\mathrm{kDa}$ heat shock protein in normal rat skin. $\mathrm{Br} \mathrm{J}$ Dermatol, 2001. 144 (2): p. 260

[9] Capon AC, Gossé AR, Iarmarcovai GN, Cornil AH, Mordon SR, Scar prevention by laser-as sisted scar healing (LASH): a pilot study using an 810-nm diode-laser system. Lasers Surg Med, 2008. 40 (7): p. 443

[10] Capon A, Iarmarcovai G, Mordon S. Laser-assisted skin healing (LASH) in hypertrophic scar revision. J Cosmet Laser Ther, 2009. 11 (4): p. 220

[11] Leclère FM, Magalon G, Philandrianos C, Unglaub F, Servell P, Mordon S. Prospective ex-vivo study on thermal effects in human skin phototypes II, IV and VI: a comparison between the 808, 1064, 1210 and 1320-nm diode laser. J Cosmet Laser Ther, 2012. 14 (1): p. 7

[12] Casanova D, Alliez A, Baptista C, Gonelli D, Lemdjadi Z, Bohbot S. A 1-Year follow-up of post-operative scars after the use of a 1210-nm laser-assisted skin healing (LASH) technology: a randomized controlled trial. Aesthetic Plast Surg, Feb 232017 